\title{
On Carbuncles and Other Excrescences
}

\author{
Philip Roe, ${ }^{*}$ Hiroaki Nishikawa, ${ }^{\dagger}$ Farzad Ismail ${ }^{\ddagger}$ and Leonardo Scalabrin $§$ \\ Department of Aerospace Engineering, University of Michigan, Ann Arbor, MI 48109-2140, USA
}

\begin{abstract}
We report observations of numerical experiments involving the carbuncle phenomenon. highlighting three distinct phases in its development. We stress that the second and third stages are logically consistent consequences of the first stage, which can largely but not wholly be explained by a one-dimensional nonlinear stability analysis. However, we do not present a cure, and are careful not to promise one, although we do feel free to criticize others.
\end{abstract}

\section{Introduction}

Shock capturing, in one form or another, has become an essential element in the prediction of aerodynamic flows, largely because the alternative of fitting the shock as a surface of discontinuity seems to be prohibitively complex in three dimensions. Shock-capturing is based theoretically on the Lax-Wendroff Theorem, ${ }^{1}$ that conservation plus stability implies convergence to a weak solution. The technology surged forward during the 1980s, largely based on use of the finite-volume technique with some form of limited reconstruction within the volumes, and a flux function or Riemann solver to compute the exchange of conserved quantities between cells. Most of the applications at that time were either to steady transonic flows in aerodynamics, or to unsteady high-energy flows in weapons simulations or astrophysics. It may be significant that much of the present unease derives from those whose interests lie in steady high-energy flows. During the time of rapid algorithm development, hypersonic research was in one of its dormant phases.

Despite the apparently secure theoretical foundations, some anomalies seem to arise from the use of shock-capturing methods. The most pervasive of these, in one form or another, is the carbuncle phenomenon It was first reported by Peery and Imlay ${ }^{2}$ that the flow past a circular cylinder. when computed by a capturing technique, did not display the expected smooth bow shock, but a rather bizarre configuration like the one shown in Figure 1. Similar behavior has since been reported by many authors. A simpler form of the anomaly was demonstrated by Quirk, ${ }^{3}$ who examined a simple one-dimensional shock propagating in a very slightly nonuniform grid; the shock broke up and took on a similar configuration. Quirk surmised that the instability was numerical, possibly an odd-even decoupling. Since then the two phenomena have gathered a large literature. Pandolfi and d'Ambrosio ${ }^{4}$ amassed a large collection of computational data for supersonic flows past circular cylinders, and concluded that 'most' shock-capturing schemes would produce a carbuncle, except for a few notoriously dissipative schemes. With some schemes, however, the phenomenon only appeared on very fine grids.

They, and other authors, have noted that the phenomenon passes all tests for a weak solution to the Euler equations. The shocks satisfy the jump conditions and are compressive, entropy-satisfying discontinuities. They resemble flows that are observed experimentally, both in two-dimensional ${ }^{5}$ and axisymmetric flows. ${ }^{6}$ However, the flows have never, to my knowledge, been observed unless provoked by some artificial feature,

\footnotetext{
*Professor and AIAA Fellow

${ }^{\dagger}$ Research Fellow and AIAA Member

$\ddagger$ PhD candidate and AIAA Student Member

$\S \mathrm{PhD}$ candidate and AIAA Student Member
} 


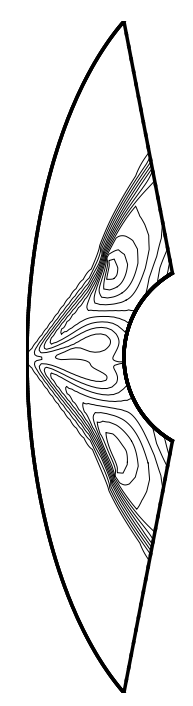

Figure 1. A typical carbuncle. The free stream is at $M=15$ with $\gamma=5 / 3$.

in two dimensions a thin plate, or in axisymmetric flow a fine spike, extending ahead of the stagnation point. Computationally, however, the occurrence is often spontaneous.

It is annoying that the phenomenon arises most readily when the flux function employed has low numerical dissipation, in particular, with schemes that are able to capture contact and shear discontinuities with minimal smearing. Liou ${ }^{7}$ has hypothesized that the problem arises with any flux function for which the mass flux across an interface depends on the pressure difference, and proposes designing schemes that suppress such a dependence. This is disconcertingly non-physical, although not necessarily inconsistent with convergence to an Euler solution. Kim et $a l^{8}$ have developed a very elaborate flux having Liou's property that $F_{1}$ does not depend on $\Delta p$. However, both they, and Liou himself, recommend adding dissipation locally to those interfaces that lie normal to the captured shock, as was also suggested by $\operatorname{Lin}^{9}$ and Sanders, Morano and Drouguet. ${ }^{10}$ If if should turn that this strategy is really necessary, we may anticipate trouble trying to compute shock/boundary-layer interactions. Moreover, nobody has yet suggested how to implement such an adaptive dissipation on unstructured grids.

An alternative path to understanding was pioneered by Robinet et al. ${ }^{11}$ They revisited the classical theory of shockwave stability, as worked out by Dyakov, ${ }^{12}$ and presented in Landau and Livshitz. ${ }^{13}$ One seeks normal modes for the subsonic flow behind a stationary normal shock, sinusoidal along the shock and exponential normal to it. This analysis will be presented below. Yet another approach was taken by Dumbser, Moschetta and Gressier ${ }^{14}$ who formulated the stability of a planar captured shock as a numerical eigenvalue problem. However, this does little more than confirm the instability of cases already known to be unstable, without supplying much insight into the mechanisms involved. In an earlier paper ${ }^{15}$ the first author speculated that since the carbuncle invariably seemed to result in spurious vorticity, a form of damping that controlled vorticity might prove effective. Such a damping has now been implemented, ${ }^{16}$ but has proved ineffective unless applied in a very heavy-handed manner. When the carbuncle wants to happen, it is very hard to stop it. It now seems wiser to hedge one's bets by declaring that there may well be more than mechanism involved. 
Rather surprisingly, there is a purely one-dimensional version of the carbuncle, involving nothing more than the one-dimensional computation of a stationary shock. It is an obvious practical requirement that the shock must be able to sit stably at any location relative to the grid. However, Barth, in $1989^{17}$ discovered that certain locations correspond to unstable equilibria, but that this only happens above a critical Mach number that depends on the ratio of specific heats (and presumably in general on the equation of state). Once again, however, the analysis is purely numerical, and affords not a lot of insight into the mechanism.

\section{Phenomenology}

We have made movie visualizations of the evolving instability of a planar shockwave on a uniform grid ${ }^{\mathrm{a}}$. Although we have tested various combinations of schemes, grids and Mach numbers, we make at present no claim to comprehensive coverage of the possibilities. Nevertheless, we are struck by a remarkable universality in our observations so far. The carbuncle invariably develops in three phases.

Pimples The first phase of instability is localized around the shock itself. Contour maps (of any flow variable) take on a spotty appearance. Exactly where the spots appear along the shock can be influenced by seeding the initial data, either with a single perturbation, a periodic perturbation, or random perturbations. This makes it plain that the phenomenon is not associated with odd-even decoupling. It can develop with any periodicity in the $\mathrm{y}$-direction. However, if you provide an odd-even seeding, then an odd-even pattern will develop.

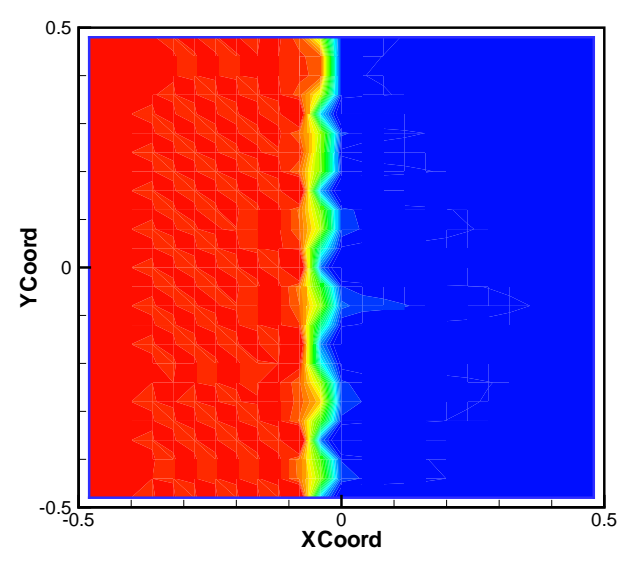

Figure 2. The initial stage of the shock instability. Colors correspond to Mach number. The Mach number ahead of the shock is 3.0, and one cell in the supersonic region was slightly perturbed.

BLEEDING The first stage can develop quite strongly before any perturbations behind the shock become strongly visible, but when they do, they correlate exactly with the locations of the pimples. Contours of velocity or Mach number show the strongest patterns, revealing parallel jets of alternating high and low velocity. Pressure perturbations are much smaller. The magnitude of these velocity disturbances grows. The faster ones become supersonic and form contracting jets, while the slower regions expand. Progressively, the slow jets first stagnate, and eventually go into reverse, penetrating through the shock to form ..

\footnotetext{
${ }^{a}$ Quirk triggered his instabilities by slightly perturbing the grid, but actually any kind of perturbation will work, and sometimes nothing seems to be needed except rounding errors
} 


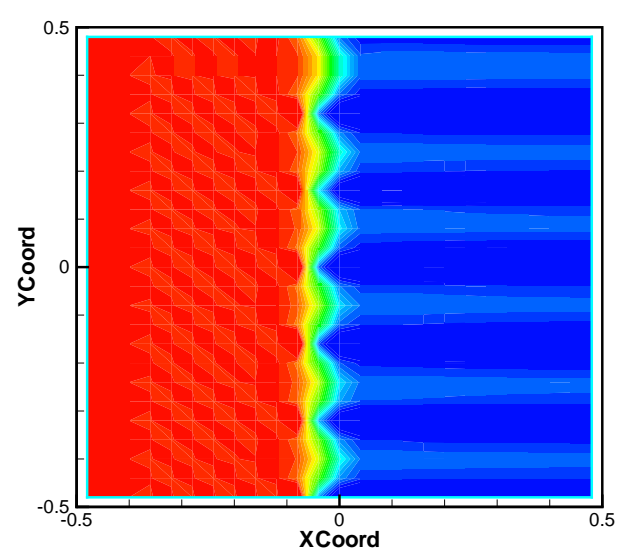

Figure 3. The second stage of the shock instability. The shock is still plane in an average sense, but a series of parallel jets develop behind it.

CARBUNCLES It usually happens that one of the reverse jets in the bleeding stage develops faster than the others, and dominates the final stage of computational evolution. A wedge-shaped region develops ahead of the shock, growing in self-similar fashion from the point of initial penetration. It is bounded by a pair of oblique shocks, which of course deflect the incoming flow, leaving a region of almost stagnant gas in the core. The angle made by these shocks is highly repeatable from run to run with given initial Mach number, and indeed does not change much when the Mach number is varied. It is tempting to say that when you have seen one carbuncle you have seen them all! In fact, results from a fully multidimensional fluctuation-splitting code gave very similar results to regular finite-volume schemes.

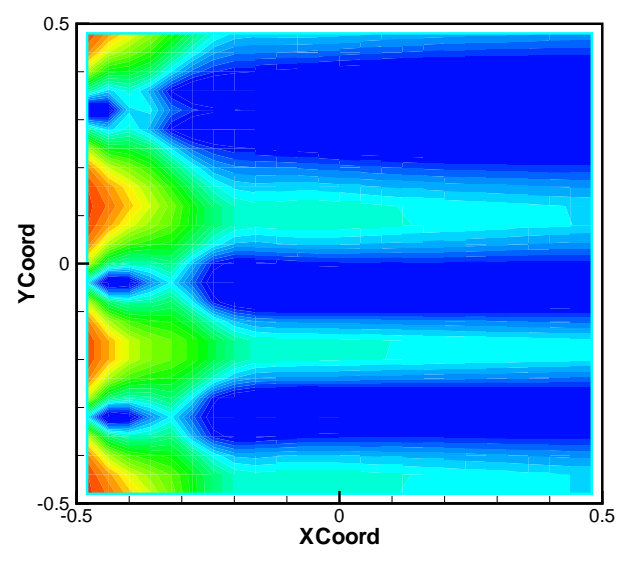

Figure 4. The third stage of the shock instability. The wedges extending into the supersonic region have developed in a self-similar fashion, but stabilize (in this case) on reaching the inflow boundary.

The boundary conditions for our simulations were simply to over specify one row of cells at inlet and one row at outlet. This is not enough to fix the shock in a particular location, so that once the instability has developed, it may begin to drift to the left or to the right. Sometimes the shock would remain center-stage 
for a long period, and the original carbuncle might collapse, to be succeeded by others.

\section{Analysis of Stage Two}

We give here an abbreviated version of the standard shock stability analysis. ${ }^{12,13}$ This begins by considering possible perturbations of the sub sonic flow behind the shock. It would not be surprising if the initial-value problem for an elliptic flow were to be unstable; it will be interesting to find the exact modality. We begin by seeking solutions to the linearized equations

$$
\mathbf{u}_{t}+\mathbf{A} \mathbf{u}_{x}+\mathbf{B} \mathbf{u}_{y}=0
$$

in the form

$$
\mathbf{u}=e^{i k y+\xi x-\omega t} \mathbf{r}, \quad \mathbf{u}=(p, u, v, s)^{T} .
$$

Under suitable nondimensionalization, we have

$$
\mathbf{A}=\left[\begin{array}{cccc}
M & 1 & 0 & 0 \\
1 & M & 0 & 0 \\
0 & 0 & M & 0 \\
0 & 0 & 0 & M
\end{array}\right], \quad \mathbf{B}=\left[\begin{array}{llll}
0 & 0 & 1 & 0 \\
0 & 0 & 0 & 0 \\
1 & 0 & 0 & 0 \\
0 & 0 & 0 & 0
\end{array}\right]
$$

and hence we require that

$$
\begin{array}{r}
\operatorname{det}\left[\begin{array}{cccc}
M \xi-\omega & \xi & k & 0 \\
\xi & M \xi-\omega & 0 & 0 \\
k & 0 & M \xi-\omega & 0 \\
0 & 0 & 0 & M \xi-\omega
\end{array}\right] \\
\left.\left.=(M \xi-\omega)^{2}\right)\left((M \xi-\omega)^{2}\right)-\xi^{2}+k^{2}\right)=0
\end{array}
$$

The repeated root $\omega=M \xi$ relates to shear and entropy disturbances; the remaining roots are related to acoustic waves. To see whether these disturbances are linearly independent, we assemble the right eigenvectors into a square matrix;

$$
\mathbf{R}=\left[\begin{array}{cccc}
\sqrt{\xi^{2}-k^{2}} & -\sqrt{\xi^{2}-k^{2}} & 0 & 0 \\
\xi & \xi & -i k & 0 \\
i k & i k & \xi & 0 \\
0 & 0 & 0 & 1
\end{array}\right]
$$

and calculate $\operatorname{det} \mathbf{R}=-2\left(k^{2}-\xi^{2}\right)^{3 / 2}$. We find that if $k=\xi$ three of the eigenvectors coincide. Rather surprisingly, the acoustic and shear disturbances are indistinguishable. This resonance creates the possibility of a non-normal mode, with associated growth and instability. The unstable modes are

$$
\mathbf{u}=e^{i k y+k(x-M t)}(0,1, i, 0)^{T}
$$

with $k$ any positive number ${ }^{\mathrm{b}}$. The modes have two interesting properties; they convect with the flow, being functions only of $x-M t$, and they are divergence free $\left(u_{x}+v_{y}=\xi r_{2}+i k r_{3}=0\right) . \mathrm{Xu}^{18}$ described this mode intuitively, but rejected it as an explanation because he felt that a plane parallel flow must be stable.

We find that these properties are shared by the second stage of our computations. Figures 5 ad 6 show, respectively, $L_{2}$ norms of the vorticity and divergence for that part of the flow in the right half of the domain. The vorticity (enstrophy) grows rapidly with the onset of Stage Two, peaking when Stage Three is reached. The divergence remains virtually flat the whole time.

\footnotetext{
${ }^{\mathrm{b}} \mathrm{It}$ is to be expected that no length scale emerges from this analysis.
} 


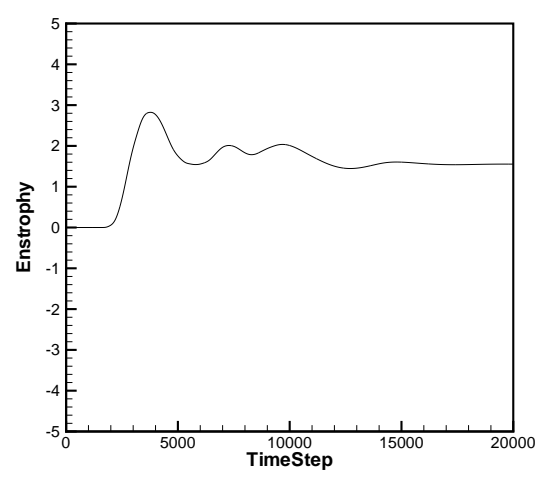

Figure 5. The $L_{2}$ norm of the vorticity "enstrophy" grows rapidly once the second stage sets in.

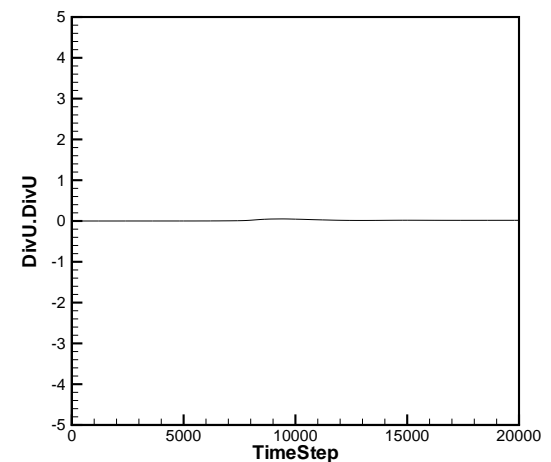

Figure 6. The $L_{2}$ norm of the divergence remains almost flat, even when the flow is violently perturbed.

\section{Analysis of Stage One}

The next step in the classical analysis of shockwave stability is to determine whether the unstable modes, now including a generalized eigenvector, are compatible with the boundary conditions on a perturbed shock. It can be shown, for a perfect gas, that they are not, and it may be concluded that the shock is stable, but the analysis is delicate, and in fact for some equations of state the opposite conclusion is reached. The next step in analyzing the carbuncle would be to see if the unstable mode is compatible with the boundary conditions imposed by a captured shock. However, this is extremely difficult, because captured shocks have a structure not describable by simple algebra. However, it is known, from a 1989 paper that was not widely read ${ }^{17}$ that $^{2}$ captured shocks have properties different in some respects from those of ideally thin discontinuities.

Consider, then, a purely one-dimensional computation of a stationary shock. The solution will be a sequence of cells, all in the state $\mathbf{u}_{L}$, followed by another sequence of cells all in the state $\mathbf{u}_{R}$ where $\mathbf{F}\left(\mathbf{u}_{R}\right)=$ $\mathbf{F}\left(\mathbf{u}_{L}\right)$. There must also be at least one cell that is in an intermediate state $\mathbf{u}_{C}$, otherwise we will not be able to accommodate the shock in a continuous manner if it makes a slow $t$ translation along the grid. The simplest case to analyze is that of one intermediate state, which is encountered for both the Godunov and Roe fluxes. Then, there exists some set of states $\mathbf{u}_{\mathbf{C}}$ including $\mathbf{u}_{L}$ and $\mathbf{u}_{R}$ as limiting cases, such that the configuration $\left.\mathbf{u}_{L}, \ldots, \mathbf{u}_{L}, \mathbf{u}_{C}, \mathbf{u}_{R}, \ldots \mathbf{u}_{R}\right)$ is in equilibrium. For the two fluxes named, this set is the set of states connected to $u_{R}$ by a reverse shock. that is the states $\mathbf{u}_{C}$ such that $\mathbf{F}\left(\mathbf{u}_{C}\right)-\mathbf{F}\left(\mathbf{u}_{R}\right)=S\left(\mathbf{u}_{C}-\mathbf{u}_{R}\right)$, with $\rho_{L} \geq \rho_{C} \geq \rho_{L}$.

Barth's under-appreciated discovery is that not all of these equilibria are stable. At sufficiently high Mach numbers, the shock is stable only at certain locations on the grid. At other locations it will spontaneously shift to a stable location ${ }^{\mathrm{c}}$ The analysis is purely numerical, but Barth shows that only four cells need to be considered, creating a system with 16 degrees of freedom. Barth then created a numerical eigenvalue problem by studying an arbitrary perturbation of the data. Some modes are always neutrally stable, because they correspond to moving from one equilibrium to another, and others are always stable. However, some modes are either stable or unstable, depending on the selection of $\mathbf{u}_{C}$, or, what amounts to the same thing, where the shock sits relative to the grid. Interestingly, for a given value of $\gamma$, the instability only occurs for Mach numbers greater than some critical value, which 'explains' why shock instability seems to be a hypersonic phenomenon.

Some indication that the algebra might, with enough effort, prove tractable comes from making a curve-fit

${ }^{\mathrm{c}} \mathrm{Q}$. Where does a Mach 20 shock wave sit? A. Any place it likes! 
to Barth's numerical results, which gives an excellent prediction of the threshold Mach number as

$$
M_{c r i t}=\frac{3-\gamma}{5 / 3-\gamma}
$$

We will return later to an interesting implication of this formula.

The idea that captured shocks can be stable or unstable depending on their location does explain some common observations. Sufficiently strong shocks often take on a rather jagged appearance, clinging closely to one grid line before suddenly jumping to the next. An example is shown in Figure 7 Sometimes the rough nature of a plot can be explained by some idiosyncracy of the plotting package, but I do not think that this is enough to explain all the cases. Clearly, such a shock structure cannot be conducive to accuracy, and the kinks may well be starting points for larger instabilities. The first author, in 1978, developing an early version of his Euler solver, studied the subsonic flow over a forward-facing step, a test case made famous by. ${ }^{19}$ The number of cells defining the front face was varied between 10 and 20, using every integer value (no upper wall was included) The convergence of the stagnation conditions to their correct values was very erratic, perhaps because the captured shock was sticking to the grid. Many papers claiming to 'cure' the carbuncle show only a few contour plots that look clean. Genuine evidence of convergence is largely missing. On unstructured grids, the shock often has a very irregular shape, but seems less likely to develop into the

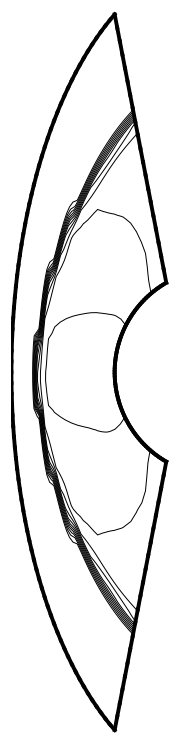

Figure 7. Flow over a cylinder at $M=15, \gamma=5 / 3$. The shock seems to 'stick' to one row of cells, before jumping to the next.

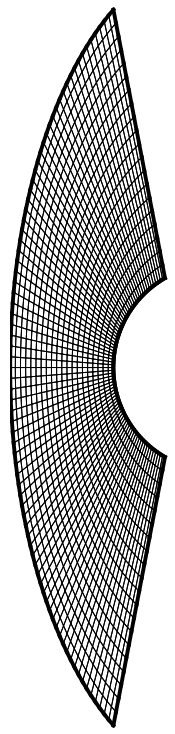

Figure 8. The grid used for the computation on the left.

full carbuncle. The quality of the results, however, can be very poor. 


\section{The Strange Case of the Monatomic Gas}

From the equation 5 it can be predicted that no one-dimensional shock instability will occur at any Mach number if $\gamma=5 / 3$ so that we deal with a monatomic gas. After becoming aware of this result, we requested Peter Gnoffo, of the NASA Langley Aerothermodynamics Branch, to conduct some tests with the LAURA code. Much to his surprise (Gnoffo, private communication) cases that had been unstable when run with $\gamma=7 / 5$ proved to be stable with $\gamma=5 / 3$. This seemed to be strong confirmation that we had a handle on the problem. However, after presenting these conclusions at a seminar in Paris, Jean-Marc Moschetta informed us that he found carbuncles in cylinder flows, even with $\gamma=5 / 3$. Subsequently, when we ran our own codes on cylinder flows rather than plane shock flows, we found the same. We also found that our experimentally-determined threshold Mach numbers were different on one-dimensional grids (where they agreed with Barth's analysis) and on two-dimensional grids. In the latter case, the threshold Mach numbers were much smaller (2.0 rather than 6.0 for $\gamma=7 / 5$ ) although somewhat greater than those reported by Robinet et al. Perhaps the difference is we did not count a case as unstable unless it went all the way to Stage Three.

The inescapable conclusion seems to be that even if one-dimensional stability is necessary, it is not sufficient. Nevertheless, our opinion is that some form of discrete shock instability is the driving mechanism for these anomalous solutions.

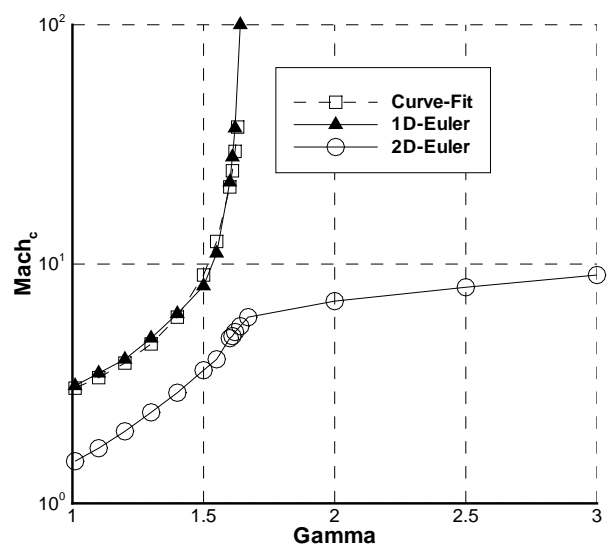

Figure 9. Threshhold Mach numbers observed experimentally for the stationary one-dimensional shock on one-or two-dimensional grids, using the first-order Roe scheme.

\section{Lax-Wendroff Theorem}

At the most theoretical level, we are left struggling to explain the discrepancy between the observations and the Lax-Wendroff theorem, implying that consistency and stability, together with an entropy condition, will yield convergence to correct weak solutions. In fact, I suspect that there are no hidden flaws in the theorem. We need to view it a little more broadly. To begin with, there is no uniqueness proof for the Euler equations, and several examples of nonuniqueness. It would be logically consistent if the theorem merely guaranteed convergence to some weak solution, with no promise that we will like it. Of course, a theoretical resolution of this kind would be small cosolation to frustrated engineers.

Also, the definition of stability in the proof is rather general, but in practice we assume that linear stability in the sense of von Neumann is sufficient. In fact as the mesh is refined, most pairs of neighbor cells 
will approach each other closely in their states, and a linear analysis will, in the limit, apparently be enough. However, pairs of cells that straddle a captured shock will never approach the same state, and a nonlinear stability analysis will be needed locally. Such an analysis is difficult, even in the scalar case. One needs some positive quantity that be guaranteed to remain bounded in exact solutions of the governing equations, and then to shown that such a result continues to hold for some choice of discretization. Almost the only candidate for this is the total entropy of the fluid ${ }^{\mathrm{d}}$ Pioneering attempts to develop a nonlinear, entropy-based theory of CFD stability were made by Merriam $^{20}$ and considerably extended by Barth. ${ }^{21}$

It is important to note that the notion of entropy here is a stronger one than that usually invoked in CFD. We commonly speak of schemes as 'entropy-satisfying' if they distinguish correctly between physical and non-physical shocks, but it is still possible that a discrete version of the Second Law of Thermodynamics may be violated locally. Both Barth ${ }^{21}$ and $^{22}$ have designed schemes that provably satisfy entropy conditions of this stronger kind. Barth (private communication) finds that his flux leads to shocks having more than one internal state, and which are stable (in the purely one-dimensional sense) at any grid location at any Mach number. However, the schemes are at present very complicated and only demonstrated on very academic problems. It is not yet known whether they can contribute to solving the carbuncle issue.

\section{Acknowledgements}

We are grateful to Dr Aldo Bonfiglioli for numerous interesting discussions during his tenure as a Fulbright Fellow in our Department. Also we thank Peter Gnoffo and Bil Kleb, NASA Langley for constant interest. The first author thanks Jack Benek and Jim Miller for valuable feedback during a visit to the Air Force Research Laboratories at Wright-Patterson Air Foce Base, Dayton, Ohio. This work was supported by the Space Vehicle Technology Institute under grant NCC3-989 jointly funded by NASA and DOD within the NASA Constellation University Institutes Project, with Claudia Meyer as the project manager.

\section{References}

${ }^{1}$ Lax, P. D., Wendroff, B, Systems of conservation laws, Comm Pure Appl Math, 13, p.217, 1960

${ }^{2}$ Peery, K.M. Imlay, S.T., Blunt body flow simulations, AIAA Paper 88-2924, 1988.

${ }^{3}$ Quirk, J.J., A contribution to the great Riemann solver debate, Int. J. Num. Meth. Fluids 18 p.555. 1994.

${ }^{4}$ Pandolfi, M., d'Ambrosio, D., Numerical instabilies in upwind methods, analysis and cures for the 'Carbuncle' phenomenon, J. Comp. Phys., 166, p.271, 2001.

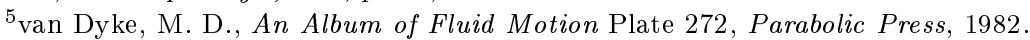

${ }^{6}$ S. Bogdonoff, I.E. Vas, Preliminary investigation of spiked bodies at supersonic speeds, J. Aero. Sci. 26. p.584, 1959.

${ }^{7}$ Liou, M. S., Mass flux schemes and connections to shock instability, J. Comp Phys., 160, p.632, 2000.

${ }^{8}$ Kim, S-s, Kim, C, Rho, O-H, Hong, S.K., Cures for the shock instability, Development of a shock-stable Roe scheme, $J$. Comp Phys., 186, p.342, 2003.

${ }^{9}$ Lin, H-C, Dissipative additions to flux-difference splitting, J. Comp Phys., 117, p.20, 1995.

${ }^{10}$ Sanders, R., Morano, E., Drouget, M. C., Multidimensional dissipation for upwind schemes, stability and applications to gasdynamics, J. Comp Phys., 145, p.511, 1998.

${ }^{11}$ Robinet, J. C., Gressier,J., Casalis, G., Moschetta, J-M., Shock wave instability and carbuncle phenomenon; same intrinsic origin J. Fluid Mech, 417, p.237, 2000.

${ }^{12}$ D'yakov, S. P. Z. Eksper. Teort. Fiz., 27, p. 288, 1954.

${ }^{13}$ Landau, L. D. , Livshitz, E. M. , Fluid Mechanics, Pergammon, 1959.

${ }^{14}$ Dumbser, M. Moschetta, J-M., Gressier, J., A matrix stability analysis of the carbuncle phenomenon, J. Comp Phys., 197, p.647, 2004.

${ }^{15}$ Roe, P. L. Capturing vorticity, AIAA-2001-2523 AIAA 16th Computational Fluid Dynamics Conference, Anaheim, CA, June 11-14, 2001

${ }^{16}$ F.Ismail, P. L. Roe, Toward a vorticity-preserving second-order finite-volumescheme solving the Euler equations, AIAA CFD meeting,Toronto, 2005.

\footnotetext{
d. Stability proofs are usually given in terms of a mathematical entropy, which is the negative of the physical entropy $S$, can
} be defined as positive and always decreases 
${ }^{17}$ Barth, T. J., Some notes on shock-resolving flux functions part I, stationary characteristics, NASA TM-101087, Ames Research Center, 1989.

${ }^{18} \mathrm{Xu}, \mathrm{K}$., Gas-kinetic schemes for unsteady compressible flow simulations, VKI Lecture series 1998-03, 1998.

${ }^{19}$ Woodward, P., Colella, P., The numerical simulation of two-dimensional fluid flow with strong shocks, J. Comp. Phys., 54, p.115, 1984

${ }^{20}$ Merriam, M. L., An entropy-based approach to nonlinear stability, NASA TM 101086, 1989.

${ }^{21}$ Barth T. J., Numerical methods for gasdynamic systems on unstructured meshes, in An Introduction to Recent Developments in Theory and Numerics of Conservation Laws, eds. Kröner, D., Ohlberger, M., Rohde, C., Springer , 1999.

${ }^{22}$ Tadmor, E., Twenty examples of entropy-stable schemes, colloquium presentation available at

www. ima . umn.edu/talks/workshops /5-11-15.2004/tadmor_Lecture_entropy_stability_20_examples .pdf 